\title{
On the Agaciro Equation via the Scope of Green Function
}

\author{
Abdon Atangana ${ }^{1}$ and Innocent Rusagara ${ }^{2}$ \\ ${ }^{1}$ Institute for Groundwater Studies, Faculty of Natural and Agricultural Sciences, University of the Free State, \\ Bloemfontein 9300, South Africa \\ ${ }^{2}$ School of Computational and Applied Mathematics, University of Witwatersrand, Private Bag 3, Wits, \\ Johannesburg 2050, South Africa \\ Correspondence should be addressed to Innocent Rusagara; rusagara2002@yahoo.fr
}

Received 18 October 2013; Accepted 20 November 2013; Published 21 January 2014

Academic Editor: Muhammet Kurulay

Copyright (C) 2014 A. Atangana and I. Rusagara. This is an open access article distributed under the Creative Commons Attribution License, which permits unrestricted use, distribution, and reproduction in any medium, provided the original work is properly cited.

We have undertaken an investigation of a kind of third-order equation called Agaciro equation within the folder of both integer and fractional order derivative. In the way of deriving the general exact solution of this equation, we employed the philosophy of the Green function together with some integral transform operators and special functions including but not limited to the Laplace, Fourier, and Mellin transform. We presented some examples of exact solution of this class of third-order equations for integer and fractional order derivative. It is important to point out that the value of Agaciro equation can be extended to describe assorted phenomenon in sciences.

\section{Introduction}

An important method used in the field of partial and ordinary linear is Green function method. It is important to remember that the construction of the Green function is an art and difficult exercise because, in the way of construction, one must first make sure of the existence and the uniqueness of this function, which is a whole topic in mathematics. Once the uniqueness and the existence are insured, one needs to have a knowledge of methods of solving either partial differential equations or ordinary differential equations, again this is a whole topic on its own in mathematics. Within the scope of fractional calculus, one further needs a clear knowledge of special function and other useful integral transform operators including but not limited to Laplace transform, Fourier transform, and Mellin transform. This method was recently extended to the scope of partial and ordinary differential equations with noninteger order derivative $[1,2]$. It should be mentioned that the finding of the Green function in the folder of the fractional calculus is very difficult because this involves at the same time some special functions and also some integral transform, for instance the Mellin, the Laplace, and the Fourier transform operators. It is perhaps important to present a brief history regarding the Green function method.

In mathematical sciences and related disciplines, a Green function is the desired answer of an inhomogeneous differential equation defined on a domain, with particular preliminary conditions or boundary conditions. By means of the superposition theory, the convolution of a Green function with a subjective function $f(x)$ on that domain is the solution to the inhomogeneous differential equation for the unknown function $f(x)$. These classes of functions are named after the British mathematician George Green, who first developed the concept in the year $1830[3,4]$. In the contemporary study of linear partial differential equations, Green's functions are revised for the most part from the standpoint of the original solutions as a replacement is significant to also draw attention to the fact that, under many-folders theories, the concept is employed in physics, more importantly in quantum field theory, aerodynamics, aeroacoustics, electrodynamics, and statistical field theory, to downgrade to an assortment of types of correlations functions, still those that do not able-bodied mathematical explanation. 
In this present work we will present the discussion underpinning the construction of Green function of a novel equation called "Agaciro" equation presented as

$$
\begin{aligned}
\partial_{x y t}^{\alpha+\beta+\gamma} & R(x, y, t)+\partial_{x t}^{\alpha+\beta} R(x, y, t)-\partial_{x y}^{\beta+\gamma} R(x, y, t) \\
& -\partial_{y t}^{\gamma+\alpha} R(x, y, t)-\partial_{x t}^{\beta+\alpha} R(x, y, t)+\partial_{t}^{\alpha} R(x, y, t) \\
& +\partial_{x}^{\beta} R(x, y, t)+\partial_{y}^{\gamma} R(x, y, t)-R(x, y, t) \\
& =f(x, y, t), \quad 0<\alpha, \beta, \gamma \leq 1 .
\end{aligned}
$$

We will examine two cases: the case where the orders of the derivative are integer numbers meaning $\alpha=\beta=\gamma=1$ and the case where $0<\alpha, \beta, \gamma<1$.

\section{Some Useful Information about Fractional Derivative}

With the purpose to provide lodgings to readers that are not in the field of fractional calculus, we dedicate this subdivision to the symposium supporting the fundamental principle of the fractional calculus. But we will much stress on the properties of the Caputo fractional derivative since it will be used throughout the remainder of the paper.

Definition 1 (Riemann-Liouville integral [5-10]). The Riemann-Liouville integral gives the orthodox outward appearance of fractional calculus. The theory for intermittent functions consequently including the "boundary condition" of repeating after a period is the Weyl integral. The RiemannLiouville integral of order $\alpha$ of a function $f(x)$ is given by

$$
{ }_{a} D_{t}^{-\alpha} f(x)={ }_{a} I_{t}^{\alpha} f(x)=\frac{1}{\Gamma(\alpha)} \int_{a}^{x}(x-t)^{\alpha-1} f(t) d t .
$$

Definition 2 (Caputo fractional derivative). There is one more option for working out the definition of fractional derivation: Caputo established it in 1967 in his paper [11]. Quite the opposite of the Riemann Liouville fractional derivative, when getting to the bottom of differential equations by means of Caputo's definition, it is not indispensable to describe the fractional order initial conditions. Caputo's definition is illustrated as follows:

$$
{ }_{0}^{C} D_{t}^{\alpha} f(x)=\frac{1}{\Gamma(n-\alpha)} \int_{a}^{x}(x-t)^{n-\alpha-1} \frac{d^{n}}{d t^{n}} f(t) d t .
$$

The above definitions are frequently used in pure and applied mathematics.

Definition 3. The Laplace transform is an extensively used integral transform in the midst of numerous applications in physics and engineering. The Laplace transform of the function $f$ is defined as follows:

$$
\mathscr{L}(f(x))(s)=\int_{0}^{\infty} e^{-s x} f(x) d x
$$

Let us observe the Laplace transform of the fractional derivative with Caputo:

$$
\begin{aligned}
& \mathscr{L}\left({ }_{0}^{C} D_{t}^{\alpha} f(x)\right)(s) \\
& \quad=s^{\alpha} F(s)-\sum_{k=0}^{n-1} s^{\alpha-k-1} f^{(k)}(0), \quad(n-1<\alpha \leq n) .
\end{aligned}
$$

The above uses the usual initial conditions or values of the functions.

Another useful property of the Caputo derivative is the following:

$$
\begin{aligned}
{ }_{a} D_{t}^{-\alpha} & {\left[{ }_{0}^{C} D_{t}^{\alpha} f(x)\right] } \\
& =f(x)-\sum_{j=1}^{n-1} \frac{f_{j}(0)}{\Gamma(\alpha-j+1)} x^{j}, \quad(n-1<\alpha \leq n),
\end{aligned}
$$

where $\Gamma$ is the gamma function defined as

$$
\Gamma(x)=\int_{0}^{\infty} t^{x-1} e^{-t} d t
$$

The Fourier transform of a function $f(x)$ of real variable $t$ is defined as

$$
(\mathscr{F} f)(p)=\int_{-\infty}^{\infty} e^{-i x p} f(x) d x
$$

The Fourier transform of a fractional derivative of order $\alpha$ is

$$
\mathscr{F}\left[{ }_{0}^{C} D_{t}^{\alpha} f(x)\right]=(i p)^{\alpha} \mathscr{F}(f)(p) .
$$

The Mellin transform of a function $f(t)$ of a real variable $t \epsilon$ $\mathbb{R}^{+}$is defined as

$$
(\mathscr{M} f)(p)=\int_{0}^{\infty} t^{p-1} f(t) d t
$$

\section{Construction of Green Function}

We will devote this section to the symposium supporting the construction of the Green function of the nonhomogeneous Agaciro equation (1); this will be achieved via some wellknown integral operator transform. The general equation under analysis here is given as

$$
\begin{aligned}
\partial_{x y t}^{\alpha+\beta+\gamma} & R(x, y, t)+\partial_{x t}^{\alpha+\beta} R(x, y, t) \\
& -\partial_{x y}^{\beta+\gamma} R(x, y, t)-\partial_{y t}^{\gamma+\alpha} R(x, y, t) \\
& -\partial_{x t}^{\beta+\alpha} R(x, y, t)+\partial_{t}^{\alpha} R(x, y, t) \\
& +\partial_{x}^{\beta} R(x, y, t)+\partial_{y}^{\gamma} R(x, y, t) \\
& -R(x, y, t)=f(x, y, t), \quad 0<\alpha, \beta, \gamma \leq 1 .
\end{aligned}
$$

3.1. Green Function for Agaciro Equation. We will present the general solution of the Agaciro equation when $\alpha=\beta=$ $\gamma=1$. To solve this equation, we make use of two integral 
transforms: in $t$-direction, we apply the Laplace transform and in $x$-y-direction, we apply the Fourier transform.

Then applying the Laplace transform on both sides of (1), we obtain the following equation:

$$
\begin{aligned}
s \partial_{x y}^{2} R_{l} & (x, y, s)-\partial_{x y}^{2} R(x, y, 0)+s \partial_{x}^{1} R_{l}(x, y, s) \\
& -\partial_{x}^{1} R(x, y, 0)-\partial_{x y}^{2} R_{l}(x, y, s)-s \partial_{y}^{1} R_{l}(x, y, s) \\
& +\partial_{y}^{1} R(x, y, 0)-s \partial_{x}^{1} R_{l}(x, y, s)+\partial_{x}^{1} R(x, y, 0) \\
& +s R_{l}(x, y, s)-R(x, y, 0)+\partial_{x}^{1} R_{l}(x, y, s) \\
& +\partial_{y}^{1} R_{l}(x, y, s)-R_{l}(x, y, s)=f_{l}(x, y, s)
\end{aligned}
$$

Now if we apply the double Fourier transform on both sides of the above equation, meaning we apply the Fourier transform in $x$ and $y$ direction, we obtain the following homogeneous equation:

$$
\begin{aligned}
& s(i p)(i q) R_{l f f}(p, q, s)-(i p)(i q) R_{f f}(p, q, 0) \\
& \quad+s(i p) R_{l f f}(p, q, s)-(i p) R_{f f}(p, q, 0) \\
& \quad-(i p)(i q) R_{f f}(p, q, s)-s(i q) R_{l f f}(p, q, s) \\
& \quad+(i q) R_{f f}(p, q, 0)-s(i p) R_{l f f}(p, q, s) \\
& \quad+s R_{l f f}(p, q, s)-R_{f f}(p, q, 0)+(i p) R_{l f f}(p, q, s) \\
& \quad+(i q) R_{l f f}(p, q, s)-R_{l f f}(p, q, s)=f_{l f f}(p, q, s) .
\end{aligned}
$$

The above equation can be converted as follows:

$$
\begin{aligned}
& R_{l f f}(p, q, s) \\
& =\frac{1}{s(i p)(i q)-s(i p)-s(i q)+s+(i p)-(i q)(i p)+(i q)-1} \\
& \quad \times\left(f_{l f f}(p, q \cdot s)+(i p)(i q) R_{f f}(p, q, 0)\right. \\
& \quad+(i p) R_{f f}(p, q, 0) \\
& \left.\quad-(i q) R_{f f}(p, q, 0)-R_{f f}(p, q, 0)\right),
\end{aligned}
$$

where $R_{l f f}(p, q, s)=\mathscr{L}(\mathscr{F}(\mathscr{F}(R(x, y, t)))), R_{f f}(p, q, 0)=$ $\mathscr{F}(\mathscr{F}(R(x, y, 0)))$.

The general Green function associated with the Agaciro equation is given as

$$
\begin{aligned}
& G(x, y, t) \\
& \begin{aligned}
=\mathscr{L}_{s}^{-1}\left(\mathscr { F } _ { p } ^ { - 1 } \left(\mathscr{F}_{q}^{-1}\right.\right. & \\
\times & (1 \times(s(i p)(i q)-s(i p)-s(i q)+s \\
& \left.\left.\left.\quad+(i p)-(i q)(i p)+(i q)-1)^{-1}\right)\right)\right) .
\end{aligned}
\end{aligned}
$$

To find the Green function, it will perhaps be important to revert the denominator of the above to the suitable form that will allow the inversion of the two operators; thus, one can see that the denominator can be factorised as follows:

$$
\begin{aligned}
& \frac{1}{s(i p)(i q)-s(i p)-s(i q)+s+(i p)-(i q)(i p)+(i q)-1} \\
& =\frac{1}{(s-1)(i p-1)(i q-1)} .
\end{aligned}
$$

With this new version in hand it is possible for us to conclude that the Green function for the Agaciro equation is given as

$$
G(x, y, t)=\exp (x+y+t) .
$$

For the sake of simplicity, let us put

$$
\begin{aligned}
& G_{1}(x, y, t)=\mathscr{L}_{s}^{-1}\left(\mathscr{F}_{p}^{-1}\left(\mathscr{F}_{q}^{-1}\left(\frac{(i p)(i q)}{(s-1)(i p-1)(i q-1)}\right)\right)\right), \\
& G_{2}(x, y, t)=\mathscr{L}_{s}^{-1}\left(\mathscr{F}_{p}^{-1}\left(\mathscr{F}_{q}^{-1}\left(\frac{-(i q)}{(s-1)(i p-1)(i q-1)}\right)\right)\right), \\
& G_{3}(x, y, t)=\mathscr{L}_{s}^{-1}\left(\mathscr{F}_{p}^{-1}\left(\mathscr{F}_{q}^{-1}\left(\frac{(i p)}{(s-1)(i p-1)(i q-1)}\right)\right)\right), \\
& G_{4}(x, y, t)=\mathscr{L}_{s}^{-1}\left(\mathscr{F}_{p}^{-1}\left(\mathscr{F}_{q}^{-1}\left(\frac{-1}{(s-1)(i p-1)(i q-1)}\right)\right)\right) .
\end{aligned}
$$

Then we observe that

$$
\mathscr{F}\left(\mathscr{F}\left(G_{1}(x, y, t)\right)\right)=(i p)(i q) \mathscr{F}(\mathscr{F}(G(x, y, t))) .
$$

Therefore, the following relationship can be established:

$$
\begin{aligned}
G_{1}(x, y, t) & =\partial_{x} \partial_{y}[G(x, y, t)], \\
G_{2}(x, y, t) & =\partial_{y}[G(x, y, t)], \\
G_{3}(x, y, t) & =\partial_{x}[G(x, y, t)], \\
G_{4}(x, y, t) & =-G(x, y, t) .
\end{aligned}
$$

With this material in hand it is now possible for us to construct the exact solution of the Agaciro equation employing the convolution theorem:

$$
\begin{array}{r}
R(x, y, t)=\int_{0}^{t} \iint_{-\infty}^{\infty} G(x-X, y-Y, t-T) \\
\times f(X, Y, T) d X d Y d T \\
+\sum_{j=1}^{4} \int_{0}^{t} \iint_{-\infty}^{\infty} G_{j}(x-X, y-Y, t-T) \\
\quad \times R(X, Y, 0) d X d Y d T .
\end{array}
$$

The above solution is the exact solution to Agaciro equation.

We will in the next subsection present some examples of exact solution of Agaciro equation.

\subsection{Exact Analytical Solution of Some Agaciro Equations}

Example 4. Let us consider the following nonhomogeneous Agaciro equation:

$$
\begin{aligned}
& \partial_{x y t}^{3} R(x, y, t)+\partial_{x t}^{2} R(x, y, t)-\partial_{x y}^{2} R(x, y, t) \\
& -\partial_{y t}^{2} R(x, y, t)-\partial_{x t}^{2} R(x, y, t)+\partial_{t}^{1} R(x, y, t) \\
& +\partial_{x}^{1} R(x, y, t)+\partial_{y}^{1} R(x, y, t)-R(x, y, t) \\
& =\frac{4 \exp \left[-t-x^{2}\right] \cos [y]}{\sqrt{\pi}}-2 \exp [-t] \cos [y] \operatorname{erf}[x] \\
& +\frac{4 \exp \left[-t-x^{2}\right] \sin [y]}{\sqrt{\pi}}-2 \exp [-t] \sin [y] \operatorname{erf}[x]
\end{aligned}
$$


with initial condition

$$
R(x, y, 0)=\cos (y) \operatorname{erf}(x) .
$$

To derive the exact solution to (22) and (23), we apply on both sides of (22) the double Fourier-Laplace operator as presented earlier, and then we obtain the desired Green function

$$
G(x, y, t)=\exp [x+y+t] .
$$

With the above Green function in hand and making use of the initial condition equation (23), we can now obtain straightforward the following solution:

$$
\begin{gathered}
R(x, y, t)=\int_{0}^{t} \iint_{-\infty}^{\infty} G(x-X, y-Y, t-T) \\
\times f(X, Y, T) d X d Y d T \\
+\sum_{j=1}^{5} \int_{0}^{t} \iint_{-\infty}^{\infty} G_{j}(x-X, y-Y, t-T) \\
\times R(X, Y, 0) d X d Y d T \\
=\cos (y) \exp (-t) \operatorname{erf}[x] .
\end{gathered}
$$

This is the exact solution of the Agaciro equation(22)

Example 5. Let us now consider the nonhomogeneous Agaciro equation given as follows:

$$
\begin{gathered}
\partial_{x y t}^{3} R(x, y, t)+\partial_{x t}^{2} R(x, y, t)-\partial_{x y}^{2} R(x, y, t) \\
-\partial_{y t}^{2} R(x, y, t)-\partial_{x t}^{2} R(x, y, t)+\partial_{t}^{\alpha} R(x, y, t) \\
+\partial_{x}^{1} R(x, y, t)+\partial_{y}^{1} R(x, y, t)-R(x, y, t) \\
=-\frac{4 \exp \left[-t-x^{2}+y\right]}{\sqrt{\pi} y}+2 \frac{\exp [y-t] \operatorname{erf}[x]}{y} \\
+\frac{4 \exp \left[-t-x^{2}\right] \exp \text { IntegralEi }[y]}{\sqrt{\pi}} \\
-2 \exp [-t] \operatorname{erf}[x] \operatorname{expIntegralEi}[y]
\end{gathered}
$$

with initial condition

$$
R(x, y, 0)=\text { expIntegralEi }[y] \operatorname{erf}[x],
$$

where expIntegralEi $[y]$ is the exponential integral defined as

$$
\text { expIntegralEi }[y]=-\int_{-y}^{\infty} \frac{e^{-x}}{x} d x,
$$

and $\operatorname{erf}[x]$ is the error function defined as

$$
\operatorname{erf}[x]=\frac{2}{\sqrt{ } \pi} \int_{0}^{x} e^{-y^{2}} d y .
$$

To obtain the exact solution to (26) and (27), we apply on both sides of (22) the double Fourier-Laplace operator as offered earlier, and then we obtain the desired Green function

$$
G(x, y, t)=\exp [x+y+t] .
$$

With the above Green function in hand and making use of the initial condition equation (27), we can now obtain straightforward the following solution:

$$
\begin{gathered}
R(x, y, t)=\int_{0}^{t} \iint_{-\infty}^{\infty} G(x-X, y-Y, t-T) \\
\times f(X, Y, T) d X d Y d T \\
+\sum_{j=1}^{5} \int_{0}^{t} \iint_{-\infty}^{\infty} G_{j}(x-X, y-Y, t-T) \\
\times R(X, Y, 0) d X d Y d T \\
=\operatorname{expIntegralEi}[y] \operatorname{erf}[x] \exp [-t] .
\end{gathered}
$$

This is the exact solution of the nonhomogeneous Agaciro equation (26).

Example 6. Let us consider the following nonlinear Agaciro equation:

$$
\begin{aligned}
& \partial_{x y t}^{3} R(x, y, t)+\partial_{x t}^{2} R(x, y, t)-\partial_{x y}^{2} R(x, y, t) \\
& -\partial_{y t}^{2} R(x, y, t)-\partial_{x t}^{2} R(x, y, t)+\partial_{t}^{\alpha} R(x, y, t) \\
& +\partial_{x}^{1} R(x, y, t)+\partial_{y}^{1} R(x, y, t)-R(x, y, t) \\
& =\frac{2 \exp [-t+y] \operatorname{Bessel} J[1, x]}{y}
\end{aligned}
$$

together with initial condition

$$
R(x, y, 0)=\operatorname{Bessel} J[1, x] \text { expIntegralEi }[y],
$$

where $\operatorname{Bessel} J[1, x]$ is the Bessel function of first kind and is defined as

$$
\text { Bessel } J[\alpha, x]=\sum_{m=0}^{\infty} \frac{(-1)^{m}}{m ! \Gamma(m+\alpha+1)}\left(\frac{x}{2}\right)^{2 m+\alpha} .
$$

To obtain the exact solution to (32) and (33), we apply on both sides of (33) the double Fourier-Laplace operator as offered earlier, and then we obtain the desired Green function

$$
G(x, y, t)=\exp [x+y+t] .
$$

With the above Green function in hand and making use of the initial condition equation (21), we can now obtain straightforward the following solution:

$$
\begin{aligned}
R(x, y, t)= & \int_{0}^{t} \iint_{-\infty}^{\infty} G(x-X, y-Y, t-T) \\
& \times f(X, Y, T) d X d Y d T \\
& +\sum_{j=1}^{5} \int_{0}^{t} \iint_{-\infty}^{\infty} G_{j}(x-X, y-Y, t-T) \\
& \times R(X, Y, 0) d X d Y d T \\
= & \text { Bessel } J[1, x] \operatorname{expIntegralEi}[y] \exp [-t] .
\end{aligned}
$$

This is the exact solution of the nonhomogeneous Agaciro equation (32). 


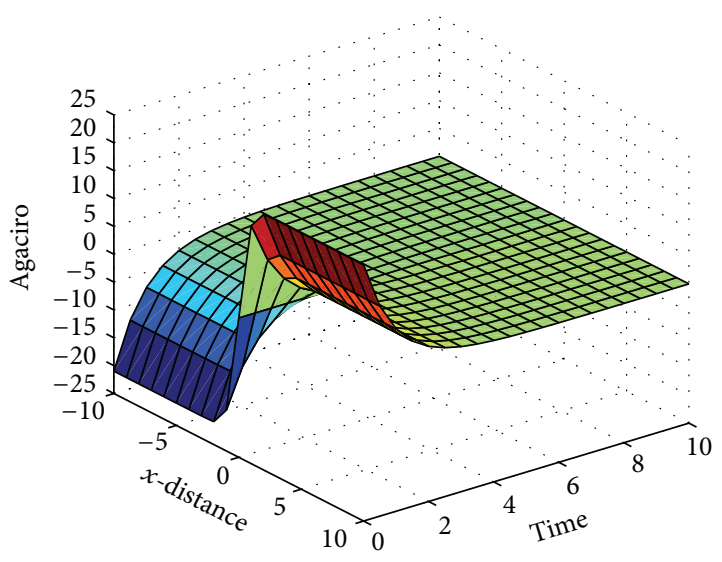

FIgURE 1: Exact solution of Agaciro equation for $y=0$.

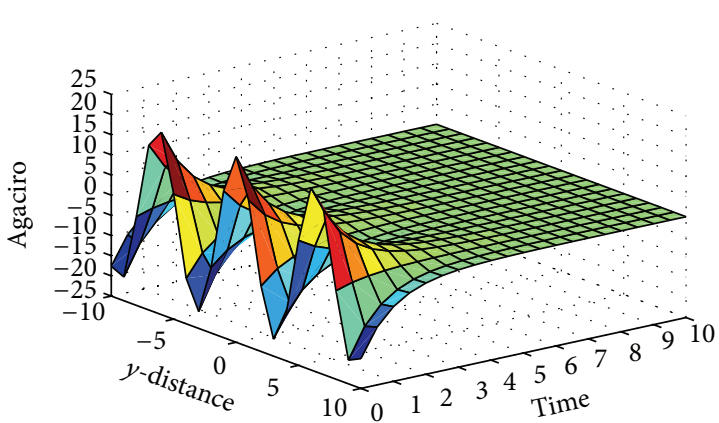

Figure 2: Exact solution of Agaciro equation (22) for $x=10$.

3.3. Analytical Results of the Agaciro Equations. We present in this section the numerical results of the nonhomogeneous Agaciro equation as function of time and space. The numerical results have been depicted in Figures 1, 2, 3, and 4.

\section{Green Function of Space-Time Fractional Agaciro Equation}

We devote this section to the symposium supporting the construction of the Green function for the space-time fractional Agaciro equation (1):

$$
\begin{aligned}
& \partial_{x y t}^{\alpha+\beta+\gamma} R(x, y, t)+\partial_{x t}^{\alpha+\beta} R(x, y, t)-\partial_{x y}^{\beta+\gamma} R(x, y, t) \\
& \quad-\partial_{y t}^{\gamma+\alpha} R(x, y, t)-\partial_{x t}^{\beta+\alpha} R(x, y, t)+\partial_{t}^{\alpha} R(x, y, t) \\
& \quad+\partial_{x}^{\beta} R(x, y, t)+\partial_{y}^{\gamma} R(x, y, t)-R(x, y, t) \\
& \quad=f(x, y, t) \quad 0<\alpha, \beta, \gamma<1 .
\end{aligned}
$$

The construction of this Green function will be achieved via the application of the double Fourier-Laplace operator as follows. Therefore applying the Laplace on both sides of (1), we arrive at the following equation:

$$
\begin{gathered}
s^{\alpha} \partial_{x y}^{\beta+\gamma} R_{l}(x, y, s)-\partial_{x y}^{\beta+\gamma} R_{l}(x, y, 0)+s^{\alpha} \partial_{x}^{\beta} R_{l}(x, y, s) \\
\quad-\partial_{x}^{\beta} R_{l}(x, y, 0)-\partial_{x y}^{\beta+\gamma} R_{l}(x, y, s)-s^{\alpha} \partial_{y}^{\gamma} R_{l}(x, y, s)
\end{gathered}
$$

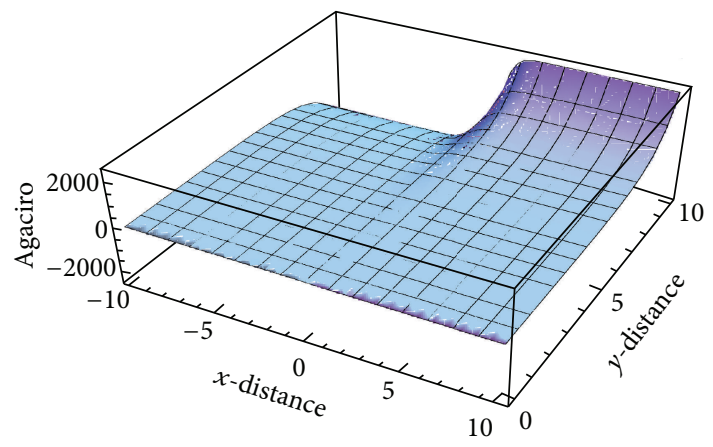

Figure 3: Exact solution of Agaciro equation (26) for $t=10$.

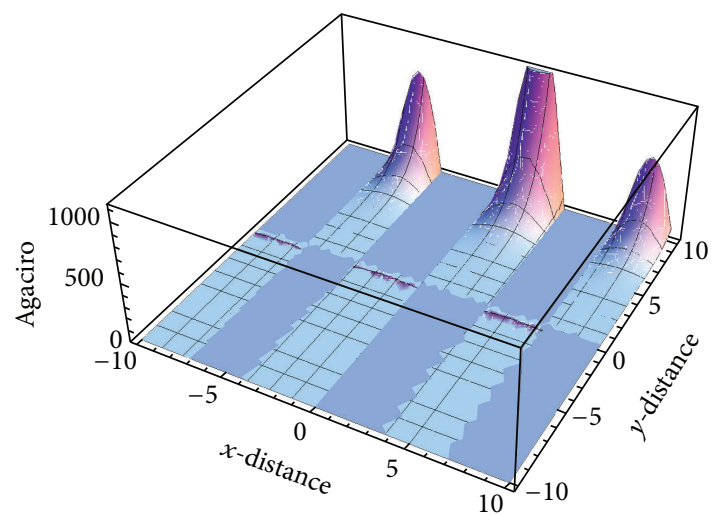

FIGURE 4: Exact solution of Agaciro equation (32) for $t=10$.

$$
\begin{aligned}
& +\partial_{y}^{\gamma} R_{l}(x, y, 0)-s^{\alpha} \partial_{x}^{\beta} R_{l}(x, y, s)+\partial_{x}^{\beta} R_{l}(x, y, 0) \\
& +s^{\alpha} R_{l}(x, y, s)-R_{l}(x, y, 0)+\partial_{x}^{\alpha} R_{l}(x, y, s) \\
& +\partial_{y}^{\beta} R_{l}(x, y, s)-R_{l}(x, y, s)=f_{l}(x, y, s) .
\end{aligned}
$$

Now if we affect the double Fourier transform on both sides of the above equation, meaning we apply the Fourier transform in $x$ and $y$ direction, we obtain the following homogeneous equation:

$$
\begin{aligned}
& s^{\alpha}(i p)^{\beta}(i q)^{\gamma} R_{l f f}(p, q, s)-s^{\alpha-1}(i p)^{\beta}(i q)^{\gamma} R_{f f}(p, q, 0) \\
& +s^{\alpha}(i p)^{\beta} R_{l f f}(p, q, s)-s^{\alpha-1}(i p)^{\beta} R_{f f}(p, q, 0) \\
& -(i p)^{\beta}(i q)^{\gamma} R_{l f f}(p, q, s)-s^{\alpha}(i q)^{\gamma} R_{l f f}(p, q, s) \\
& +s^{\alpha-1}(i q)^{\gamma} R_{f f}(p, q, 0)-s^{\alpha}(i p)^{\beta} R_{l f f}(p, q, s) \\
& +s^{\alpha-1}(i p)^{\beta} R_{f f}(p, q, 0)+s^{\alpha} R_{l f f}(p, q, s) \\
& -s^{\alpha-1} R_{f f}(p, q, 0)+(i p)^{\beta} R_{l f f}(p, q, s) \\
& +(i q)^{\gamma} R_{l f f}(p, q, s)-R_{l f f}(p, q, s)=f_{l f f}(p, q, s) .
\end{aligned}
$$


The above equation can be transformed as follows:

$$
\begin{aligned}
& R_{l f f}(p, q, s) \\
& =1 \times\left(s^{\alpha}(i p)^{\beta}(i q)^{\gamma}-s^{\alpha}(i p)^{\beta}-s^{\alpha}(i q)^{\gamma}\right. \\
& \left.\quad+s^{\alpha}+(i p)^{\beta}-(i q)^{\gamma}(i p)^{\beta}+(i q)^{\gamma}-1\right)^{-1} \\
& \times\left(f_{l f f}(p, q \cdot s)+(i p)^{\beta}(i q)^{\gamma} s^{\alpha-1} R_{f f}(p, q, 0)\right. \\
& \quad+(i p)^{\beta} s^{\alpha-1} R_{f f}(p, q, 0)-(i q)^{\gamma} s^{\alpha-1} R_{f f}(p, q, 0) \\
& \left.\quad-s^{\alpha-1} R_{f f}(p, q, 0)\right) .
\end{aligned}
$$

The broad-spectrum fractional Green function that connects to the space-time fractional Agaciro equation is provided as

$$
\begin{aligned}
& G_{\alpha \beta \gamma}(x, y, t) \\
& =\mathscr{L}_{s}^{-1}\left(\mathscr{F}_{p}^{-1}\right. \\
& \quad \times\left(\mathscr { F } _ { q } ^ { - 1 } \left(1 \left(s^{\alpha}(i p)^{\beta}(i q)^{\gamma}-s^{\alpha}(i p)^{\beta}-s^{\alpha}(i q)^{\gamma}\right.\right.\right. \\
& \quad+s^{\alpha}+(i p)^{\beta}-(i q)^{\gamma}(i p)^{\beta} \\
& \left.\left.\left.\left.\quad+(i q)^{\gamma}-1\right)^{-1}\right)\right)\right) .
\end{aligned}
$$

It is perhaps important to point out that the below equation is the fractional characteristic equation associate to the spacetime fractional Agaciro equation:

$$
\begin{aligned}
& s^{\alpha}(i p)^{\beta}(i q)^{\gamma}-s^{\alpha}(i p)^{\beta}-s^{\alpha}(i q)^{\gamma} \\
& \quad+s^{\alpha}+(i p)^{\beta}-(i q)^{\gamma}(i p)^{\beta}+(i q)^{\gamma}-1 .
\end{aligned}
$$

To discover the Green function, it will conceivably relapse the above fractional polynomial equation to the appropriate form that will consent to the inversion of the two operators; consequently, one can see that the denominator can be factorised as follows:

$$
\begin{aligned}
1 \times & \left(s^{\alpha}(i p)^{\beta}(i q)^{\gamma}-s^{\alpha}(i p)^{\beta}-s^{\alpha}(i q)^{\gamma}\right. \\
& \left.+s^{\alpha}+(i p)^{\beta}-(i q)^{\gamma}(i p)^{\alpha}+(i q)^{\gamma}-1\right)^{-1} \\
= & 1 \times\left(\left(s^{\alpha}-1\right)\left((i p)^{\beta}-1\right)\left((i q)^{\gamma}-1\right)\right)^{-1} .
\end{aligned}
$$

In order to find the inverse of the above equation, we will first accommodate readers that are not used to relationships between some special functions and some properties of Fourier and Laplace transforms. An important special function in the field of fractional calculus is the Mittag-Leffler function which is regarded as the generalized exponential function and is defined as

$$
E_{\alpha}[z]=\sum_{n=0}^{\infty} \frac{z^{n}}{\Gamma[\alpha n+1]}, \quad(z \in \mathbb{C} ; \operatorname{Re}[\alpha]>0) .
$$

The Laplace transform of the above function is given as

$$
\mathscr{L}\left(E_{\alpha}[\tau z]\right)(s)=\frac{s^{\alpha-1}}{s^{\alpha}-\tau}, \quad R(s)>0, \tau \in \mathbb{C} ;\left|\tau s^{-\alpha}\right|<1 .
$$

Another useful relation here is the following:

$$
\begin{gathered}
\mathscr{L}\left(z^{\beta-1} E_{\alpha}[\tau z]\right)(s)=\frac{s^{\alpha-\beta}}{s^{\alpha}-\tau}, \\
R(s)>0, \quad \tau \in \mathbb{C} ; \quad\left|\tau s^{-\alpha}\right|<1 .
\end{gathered}
$$

If we assume from the above equation that $\alpha=\beta$, then we obtain the following useful relationship:

$$
\begin{aligned}
& \mathscr{L}\left(z^{\alpha-1} E_{\alpha}[\tau z]\right)(s)=\frac{1}{s^{\alpha}-\tau}, \\
& R(s)>0, \quad \tau \in \mathbb{C} ; \quad\left|\tau s^{-\alpha}\right|<1 .
\end{aligned}
$$

With this new version in hand together with the above useful properties, it will be possible for us to conclude that the Green function for the space-time fractional Agaciro equation is given as

$$
G_{\alpha \beta \gamma}(x, y, t)=t^{\alpha-1} E_{\alpha}(t) x^{\beta-1} E_{\beta}(x) y^{\gamma-1} E_{\gamma}(y) .
$$

Now to have a clear relationship between the inverse of the fractional Green function and the remaining terms, we let

$$
\begin{aligned}
& G_{1} \alpha \beta \gamma(x, y, t) \\
& =\mathscr{L}_{s}^{-1}\left(\mathscr{F}_{p}^{-1}\right. \\
& \quad \times\left(\mathscr { F } _ { q } ^ { - 1 } \left((i p)^{\beta}(i q)^{\gamma} s^{\alpha-1}\right.\right. \\
& \left.\left.\left.\quad \times\left(\left(s^{\alpha}-1\right)\left((i p)^{\beta}-1\right)\left((i q)^{\gamma}-1\right)\right)^{-1}\right)\right)\right),
\end{aligned}
$$

$G_{2} \alpha \beta \gamma(x, y, t)$

$=\mathscr{L}_{s}^{-1}\left(\mathscr{F}_{p}^{-1}\right.$

$$
\begin{aligned}
\times\left(\mathscr{F}_{q}^{-1}\right. & \left(-(i q)^{\gamma} s^{\alpha-1}\right. \\
& \left.\left.\left.\times\left(\left(s^{\alpha}-1\right)\left((i p)^{\beta}-1\right)\left((i q)^{\gamma}-1\right)\right)^{-1}\right)\right)\right),
\end{aligned}
$$

$G_{3} \alpha \beta \gamma(x, y, t)$

$$
\begin{aligned}
& =\mathscr{L}_{s}^{-1}\left(\mathscr{F}_{p}^{-1}\right. \\
& \quad \times\left(\mathscr { F } _ { q } ^ { - 1 } \left((i p)^{\beta} s^{\alpha-1}\right.\right. \\
& \left.\left.\left.\quad \times\left(\left(s^{\alpha}-1\right)\left((i p)^{\beta}-1\right)\left((i q)^{\gamma}-1\right)\right)^{-1}\right)\right)\right), \\
& G_{4 \alpha \beta \gamma}(x, y, t) \quad-1 \\
& =\mathscr{L}_{s}^{-1}\left(\mathscr{F}_{p}^{-1}\left(\mathscr{F}_{q}^{-1}\left(\frac{-1}{(s-1)(i p-1)(i q-1)}\right)\right)\right) \\
& =-G_{\alpha \beta \gamma}(x, y, t) .
\end{aligned}
$$


With these functions together with the fractional Green function, we can further derive the exact solution of the class of fractional partial differential equation as

$$
\begin{gathered}
R(x, y, t)=\int_{0}^{t} \iint_{-\infty}^{\infty} G_{\alpha \beta \gamma}(x-X, y-Y, t-T) \\
\times f(X, Y, T) d X d Y d T \\
+\sum_{j=1}^{5} \int_{0}^{t} \iint_{-\infty}^{\infty} G_{j \alpha \beta \gamma}(x-X, y-Y, t-T) \\
\times R(X, Y, 0) d X d Y d T .
\end{gathered}
$$

Example 7. Consider the following time-space fractional Agaciro equation:

$$
\begin{aligned}
& \partial_{x y t}^{\alpha+\beta+\gamma} R(x, y, t)+\partial_{x t}^{\alpha+\beta} R(x, y, t)-\partial_{x y}^{\beta+\gamma} R(x, y, t) \\
& \quad-\partial_{y t}^{\gamma+\alpha} R(x, y, t)-\partial_{x t}^{\beta+\alpha} R(x, y, t)+\partial_{t}^{\alpha} R(x, y, t) \\
& \quad+\partial_{x}^{\beta} R(x, y, t)+\partial_{y}^{\gamma} R(x, y, t)-R(x, y, t) \\
& \quad=\exp [x+y+t], \quad 0<\alpha, \beta, \gamma<1,
\end{aligned}
$$

with initial condition

$$
R(x, y, 0)=0
$$

Now to solve the above equation, we follow the discussion presented earlier to obtain the desired Green function

$$
G_{\alpha \beta \gamma}(x, y, t)=t^{\alpha-1} E_{\alpha}(t) x^{\beta-1} E_{\beta}(x) y^{\gamma-1} E_{\gamma}(y) .
$$

Now using the convolution theorem together with the initial condition, we obtain the exact solution of (51) as

$$
\begin{aligned}
R(x, y, t)=\int_{0}^{t} \iint_{-\infty}^{\infty} G_{\alpha \beta \gamma}(x-X, y-Y, t-T) \\
\\
\times f(X, Y, T) d X d Y d T,
\end{aligned}
$$

since the convolution is commutative, we can reformulate the above equation as

$$
\begin{gathered}
R(x, y, t)=\int_{0}^{t} \int_{0}^{x} \int_{0}^{y} G_{\alpha \beta \gamma}(X, Y, T) \\
\quad \times f(x-X, y-Y, t-T) d X d Y d T \\
R(x, y, t)=\int_{0}^{t} \int_{0}^{x} \int_{0}^{y} T^{\alpha-1} E_{\alpha}(T) X^{\beta-1} E_{\beta}(X) Y^{\gamma-1} E_{\gamma}(Y) \\
\quad \times \exp (x-X) \exp (y-Y) \\
\times \exp (t-T) d X d Y d T .
\end{gathered}
$$

Now using the notation of the Mittag-Leffler function given in (44), we can reformulate the above equation as follows:

$$
\begin{aligned}
R(x, y, t)= & \exp (x+y+t) \\
& \times \int_{0}^{t} \sum_{n=0}^{\infty} \frac{(-T)^{n+\alpha-1}}{\Gamma(\alpha n+1)} \exp (-T) d T \\
& \times \int_{0}^{x} \sum_{k=0}^{\infty} \frac{(-X)^{k+\beta-1}}{\Gamma(\beta k+1)} \exp (-X) d X \\
& \times \int_{0}^{x} \sum_{m=0}^{\infty} \frac{(-Y)^{m+\gamma-1}}{\Gamma(\gamma m+1)} \exp (-Y) d Y .
\end{aligned}
$$

Integrating the above equation we obtain the following exact solution of the space-time Agaciro equation (51) as

$$
\begin{aligned}
R(x, y, t) & \\
=\exp (x+y+t) \sum_{n=0}^{\infty} \sum_{m=0}^{\infty} \sum_{k=0}^{\infty} & (-1)^{n+\alpha} \\
& \times(-1)^{k+1}(-1)^{m+1} \\
& \times[-\Gamma[n+\alpha]+\Gamma[n+\alpha, t]] \\
& \times[-\Gamma[k+\beta]+\Gamma[k+\beta, x]] \\
& \times[-\Gamma[m+\gamma]+\Gamma[m+\gamma, y]]
\end{aligned}
$$

\section{Conclusion}

The aim of this work was to investigate a class of partial differential equations within the concept of integer and fractional order derivative. This class of equations is referred to as Agaciro equations. We presented the general solution together with some examples of this equation within the scope of ordinary and fractional derivation. To achieve this, we made use of the so-called Green function method together with some well-known integral transform operators.

\section{Conflict of Interests}

The authors declare that there is no conflict of interests regarding the publication of this paper.

\section{References}

[1] I. Podlubny, Fractional Differential Equations, vol. 198 of Mathematics in Science and Engineering, Academic Press, San Diego, Calif, USA, 1999.

[2] K. S. Miller and B. Ross, An Introduction to the Fractional Calculus and Fractional Differential Equations, A Wiley-Interscience, John Wiley \& Sons, New York, NY, USA, 1993.

[3] Ş. S. Bayin, Mathematical Methods in Science and Engineering, chapter 18-19, John Wiley \& Sons, Hoboken, NJ, USA, 2006.

[4] A. D. Polyanin and V. F. Zaitsev, Handbook of Exact Solutions for Ordinary Differential Equations, Chapman \& Hall/CRC Press, Boca Raton, Fla, USA, 2nd edition, 2003.

[5] S. G. Samko, A. A. Kilbas, and O. I. Marichev, Fractional Integrals and Derivatives: Theory and Applications, Gordon and Breach, Yverdon, Switzerland, 1993.

[6] I. Podlubny, "Geometric and physical interpretation of fractional integration and fractional differentiation," Fractional Calculus \& Applied Analysis, vol. 5, no. 4, pp. 367-386, 2002.

[7] A. A. Kilbas, H. M. Srivastava, and J. J. Trujillo, Theory and Applications of Fractional Differential Equations, vol. 204 of NorthHolland Mathematics Studies, Elsevier Science, Amsterdam, The Netherlands, 2006.

[8] A. Atangana and A. Secer, "A note on fractional order derivatives and table of fractional derivatives of some special functions," Abstract and Applied Analysis, vol. 2013, Article ID 279681, 8 pages, 2013. 
[9] A. Anatoly, J. Juan, and M. S. Hari, Theory and Application of Fractional Differential Equations, vol. 204 of North-Holland Mathematics Studies, Elsevier, Amsterdam, The Netherlands, 2006.

[10] Y. Luchko and R. Groneflo, The Initial Value Problem for Some Fractional Differential Equations with the Caputo Derivative, Preprint Series A08-98, Fachbereich Mathematik und Informatik, Freic Universität, Berlin, Germany, 1998.

[11] M. Caputo, "Linear models of dissipation whose $Q$ is almost frequency independent-II," Geophysical Journal International, vol. 13 , no. 5, pp. 529-539, 1967. 




Advances in

Operations Research

mansans

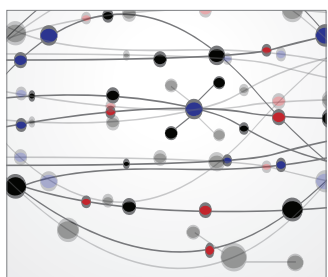

The Scientific World Journal
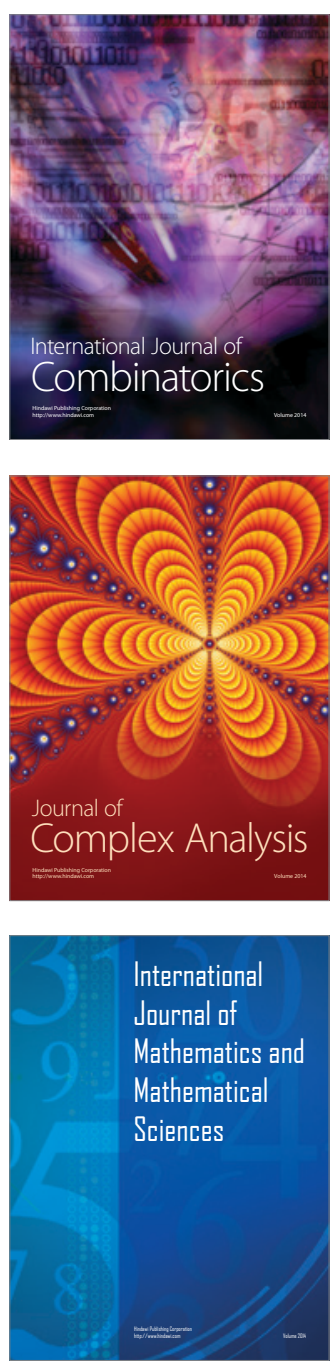
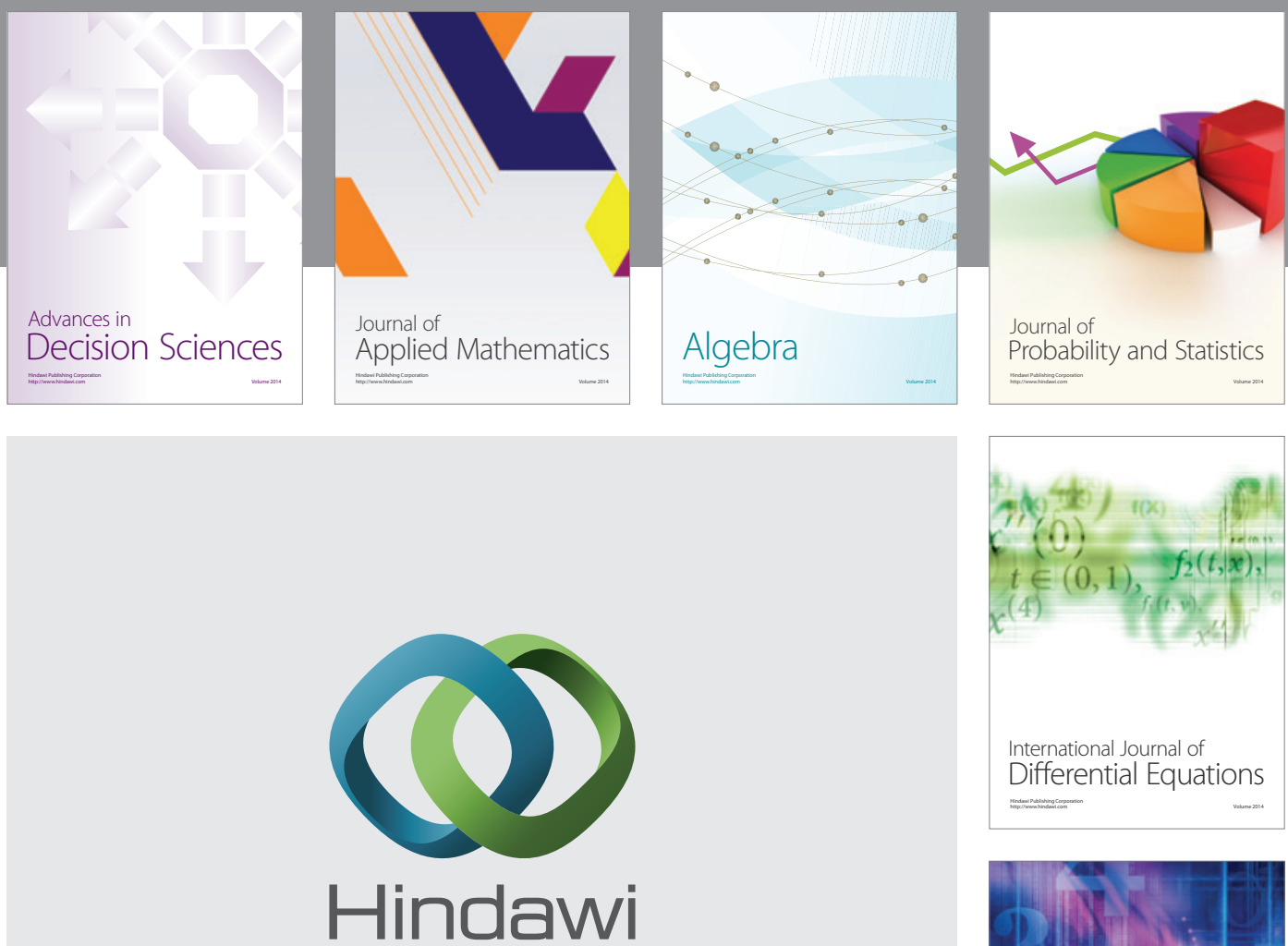

Submit your manuscripts at http://www.hindawi.com
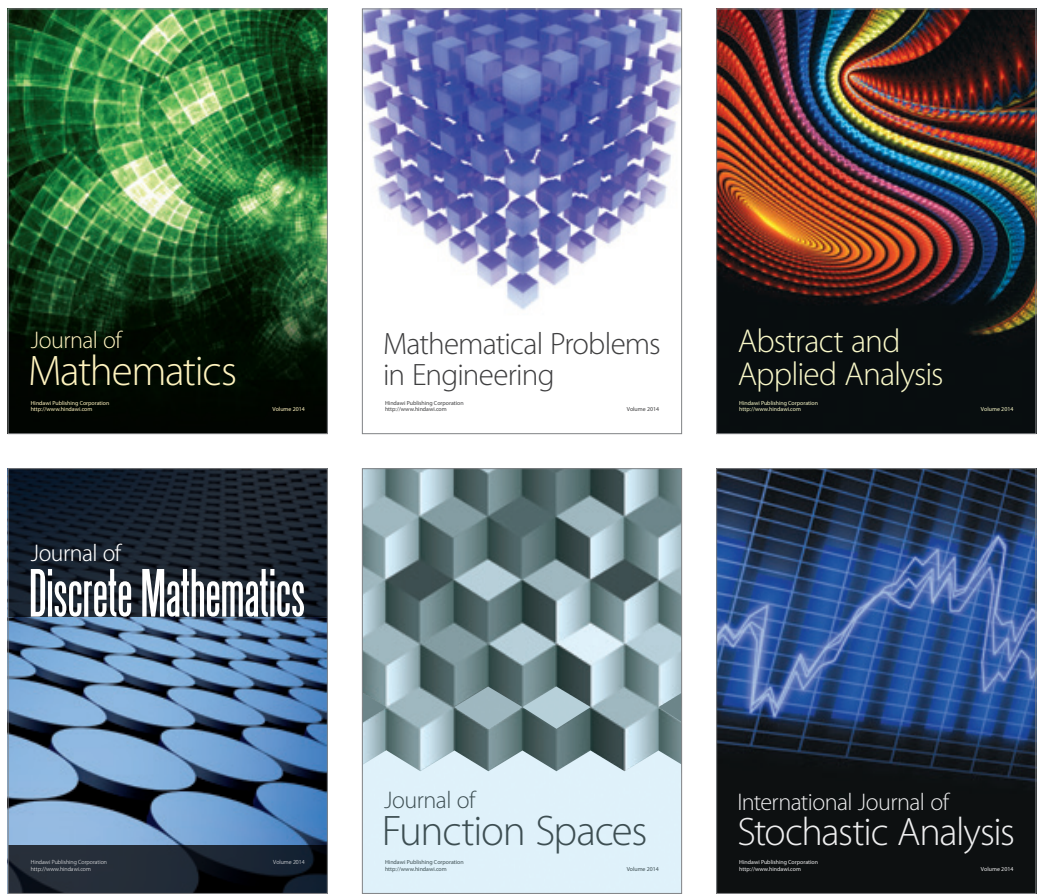

Journal of

Function Spaces

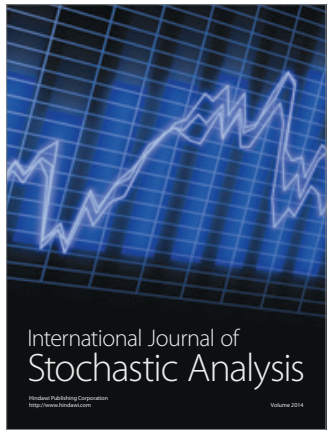


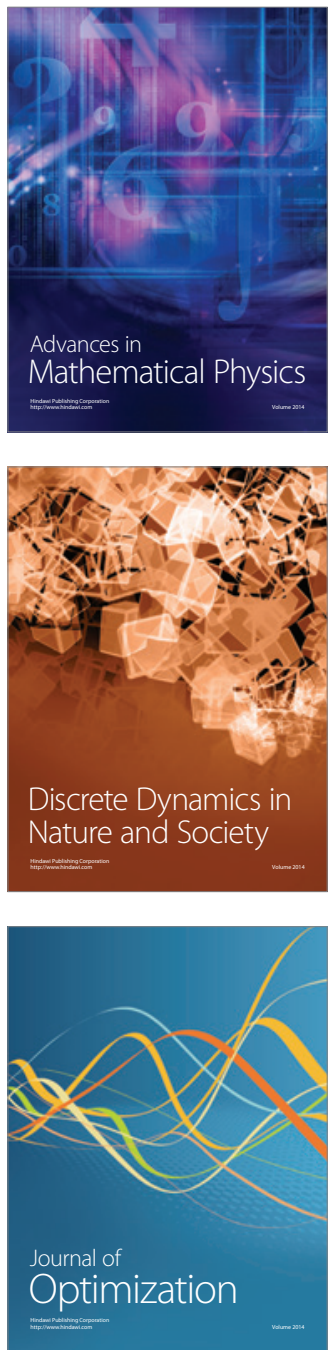